\title{
Demographic Characteristics and Clinical Features of Fibromyalgia in China: A Cross-Sectional Study
}

\author{
Juan Jiao · Zengyu Cheng · Wen Wang $\cdot$ Yayun Zhao • \\ Quan Jiang (1D
}

Received: February 1, 2021 / Accepted: March 18, 2021 / Published online: April 4, 2021

(C) The Author(s) 2021, corrected publication 2021

\begin{abstract}
Objective: The aim of this study is to characterize the demographics, fibromyalgia-related symptom severity and quality of life (QOL) among Chinese fibromyalgia patients.

Methods: A total of 124 patients who met the ACR 1990 criteria were recruited. Each subject completed a packet of questionnaires for collecting data on the demographics, medical history and severity of six major symptoms of fibromyalgia (i.e., pain, fatigue, sleep quality, depression, stress and QOL).

Results: The majority of the study subjects were women $(86.3 \%)$, were married $(78.2 \%)$ and
\end{abstract}

J. Jiao $\cdot$ Z. Cheng $\cdot$ Q. Jiang ( $₫)$

Rheumatology Department, Guang'anmen

Hospital, China Academy of Chinese Medical

Sciences, No. 5 Beixiange Street, Beijing 100053,

Xicheng District, China

e-mail: jiang.quan@hotmail.com

W. Wang

Rehabilitation Department, Shenzhen Hyzhen

Hospital, Shenzhen, China

Y. Zhao

Graduate School, Hebei University of Chinese

Medicine, Hebei, China

Y. Zhao

Rheumatology Department, Hebei Hospital of

Traditional Chinese Medicine, Hebei, China had a mean age of 49.4 years and a median symptom duration of 24 months. Compared to their female counterparts, male patients were younger, were more likely to be employed, had a higher income and were more likely to be current smokers and alcohol drinkers (all $p \leqq$ 0.027). Most patients experienced moderate to severe pain $(69.4 \%)$, severe fatigue $(70.2 \%)$ and moderate to severe depression (53.3\%). A small percentage $(19.4 \%)$ of the patients had very poor quality of sleep, and over one third (37.1\%) suffered from moderate to severe stress. Less than one third (27.5\%) patients' health status was moderately or highly impacted by fibromyalgia. The mental health of men was affected significantly more than that of women, with lower SF-36 scores in the SF-36 mental component summary $(p=0.043)$ and role emotional $(p=0.006)$.

Conclusion: This study has revealed that Chinese fibromyalgia patients might share similar demographics but milder fibromyalgia-related symptoms and better mental QOL compared to patients of other races and ethnic groups. Some differences between male and female patients were found, too. Further cross-sectional studies with a larger sample size and nationwide study sites are needed to replicate those clinical findings.

Keywords: Chinese population; Characteristics; Features; Fibromyalgia 


\section{Key Summary Points}

\section{Why carry out this study?}

Available studies indicate the prevalence of fibromyalgia in Mainland China ranges from $0.03 \%$ to $0.12 \%$, much lower than that of other countries in the world. The reason(s) for such low prevalence are largely unknown and need to be explored

Different clinical manifestations of fibromyalgia in Chinese populations might be one of the reasons for this low rate and need to be investigated

The clinical features and severity of fibromyalgia in Chinese patients are not clear because few studies have been conducted

\section{What was learned from the study?}

The study found that (1) Chinese patients have similar clinical characteristics of fibromyalgia as those of patients from other countries. (2) Chinese patients' symptoms are likely to be less severe and their quality of life might be less impacted by having fibromyalgia. The latter might contribute to the low fibromyalgia prevalence found in China if using current diagnostic methods

In addition to this discovery, further study to validate these findings and to investigate the factors that lessen the symptom severity in Chinese patients might shed some light on developing new interventions to manage this disease

\section{DIGITAL FEATURES}

This article is published with digital features, including a summary slide, to facilitate understanding of the article. To view digital features for this article go to: https://doi.org/10.6084/ m9.figshare.14224397.

\section{INTRODUCTION}

Fibromyalgia is a debilitating chronic pain disorder. In addition to the widespread body pain, fibromyalgia patients also have a cluster of other core symptoms, including fatigue, sleep disturbance, depression, anxiety, reduced physical capacity and cognitive dysfunction [1]. Fibromyalgia affects up to $5.0 \%$ of the general population worldwide and is readily recognized and treated in western and developed countries [2]. In China, the prevalence of fibromyalgia in a Hong Kong Chinese population was estimated to be $0.82 \%$ of the population using 1990 American College of Rheumatology (ACR) classification criteria for fibromyalgia [3]. Applying this estimated prevalence to China's 1.4 billion population suggests that fibromyalgia could affect as many as 11.5 million Chinese people.

However, fibromyalgia is rarely diagnosed in Mainland China, and the clinical features of Chinese fibromyalgia patients are not clear. To date, three small-scale epidemiological studies of rheumatological diseases have been conducted in two provinces in Mainland China and reported that fibromyalgia prevalence ranged from $0.03 \%$ to $0.12 \%$ using the ACR 1990 criteria [4-6]. When comparing their findings on fibromyalgia prevalence in China to other nations, even to other Asian countries like Korea (2.2\%) [7] or Japan (2.1\%) [8], this low prevalence of fibromyalgia in China raises questions about whether fibromyalgia is manifested differently in Chinese patients from other cultural and ethnic groups or if it may be underrecognized and underdiagnosed.

To study the impact of fibromyalgia in Chinese people in Mainland China, the objective of this study was to thoroughly document the demographic characteristics and clinical features, fibromyalgia-related symptom severity and quality of life (QOL) of Chinese fibromyalgia patients diagnosed using the ACR 1990 criteria. 


\section{METHODS}

\section{Patient Selection}

This was a cross-sectional study conducted between May 5, 2015, and March 31, 2016, in an outpatient rheumatology clinic at Guang'anmen Hospital, a tertiary academic hospital in Beijing, China. The study was registered with Clinical Trials.gov (identifier: NCT02449395), and the study protocol was approved by the Ethics Committee of Guang'anmen Hospital (approval no. 2015EC044). The ACR 1990 criteria [9] were used for the diagnosis of fibromyalgia in this study. Fibromyalgia secondary to other medical conditions was excluded from the study.

Study protocol: Patients visiting the outpatient clinic for widespread pain were first screened for the diagnosis of fibromyalgia using the ACR 1990 criteria. The prospective study subjects who met inclusion criteria were given a case book to complete after providing informed consent for the study. The case book includes two sections: the first section asks for patients' demographic information and medical history, and the second section contains a set of questionnaires that evaluate the symptoms of fibromyalgia and a patient self-report for the assessment of fibromyalgia based on the ACR 2010 criteria [10] (see below). One designated researcher was available on site in case the subjects had questions about the questionnaires. All patients participated in the study voluntarily. No financial compensation was offered for completing the questionnaires.

\section{Questionnaires}

All of the questionnaires used to document the symptoms of fibromyalgia in this study were translated to Chinese language, and most of them were validated as indicated in the text below.

1. Pain: pain visual analog scale (Pain VAS) and the numerical rating scale (NRS) for pain

To document the perceived severity of body pain, two self-assessment instruments were used: Pain VAS and NRS for pain. The Pain VAS is a subjective tool for the measurement of perceived average pain severity over the past week [11]. It consists of a continuous 100-mm-long line, with two endpoints, labeled "no pain" on the left and "worst possible pain" on the right.

The NRS for pain allows a person to specify the intensity of his/her pain during the previous week. It is numbered from 0 to 10 , in which 0 indicates no pain; $1-3$, mild pain; $4-6$, moderate pain; 7-9, severe pain; 10, the most severe pain possible [12].

2. Fatigue: Multidimensional Assessment of Fatigue (MAF) and Global Fatigue Index (GFI)

Fatigue was evaluated with the MAF questionnaire, a self-report assessment tool designed to measure five dimensions of fatigue: degree, severity, distress, impact on daily living and timing in the week before [13]. GFI then quantifies the five dimensions of fatigue from the MAF into one score, ranging from 0 to 50 points; a higher score indicates more severe fatigue. There are no set cut-off scores on the MAFGFI to define the grade of fatigue. An MAFGFI score of 30 was once used to define clinically severe fatigue according to a previous study [14].

3. Sleep Quality: Pittsburgh Sleep Quality Index (PSQI)

The Pittsburgh Sleep Quality Index (PSQI) assesses sleep quality during the previous month. The Chinese version of the PSQI showed good internal consistency, testretest reliability and high conceiving validity in 1996 [15]. The score of PSQI ranges from 0 to 21, with higher scores representing more severely compromised sleep quality. The ranges for the score are defined as: 0-5 points, very little compromised sleep quality; 6-10, good sleep quality; 11-15 points, fair sleep quality; $16-21$ points, very poor sleep quality [15].

4. Depression: Beck Depression Inventory-II (BDI-II)

The Chinese version of the BDI-II was used to assess symptoms of depression and 
provides a choice of four responses per question, with scores ranging from 0 to 39 points, with higher scores reflecting more severe depression [16]. The cut-off points for scores are: 0-4 points, no relevant depressive symptoms; 5-7 points, mild depressive symptoms; 8-15 points, moderate depressive symptoms; $16-39$ points, severe depressive symptoms. This Chinese version was assessed for reliability and validity in patients with major depression in 2009 [16].

5. Stress: the Perceived Stress Scale (PSS) The Perceived Stress Scale (PSS) is a psychological instrument designed to measure the level of perceived stress, and its Chinese version was validated in 2003 by studying Chinese urban residents' perceptions of urban transformation [17]. The score ranges from 0 to 56 points, with higher scores corresponding to higher perceived stress, and the cut-off points are defined as below: 0-28 points, a perception of mild stress; 29-42 points, moderately severe stress; 43-56 points, severe stress [17].

6. Health-related QOL: The Medical Outcome Study Short Form (SF-36)

The SF-36 evaluated eight domains of health-related QOL: physical functioning, role physical, bodily pain, general health, vitality, social functioning, role emotional and mental health index. From the SF-36, two distinct component scores were derived: the physical component summary (SF-36 PCS) and the mental component summary (SF-36 MCS). Both SF-36 PCS and SF-36 MCS range from 0 to 100; higher scores indicate better health-related QOL [18]. The Chinese version of the SF-36 was tested and validated in 2002 [18].

7. Fibromyalgia impact: Fibromyalgia Impact Questionnaire (FIQ)

The FIQ has been validated as an effective tool for assessing the effect of fibromyalgia on the health status of the patients [19]. It contains 20 questions to assess 10 domains of the health status over the last week, including: physical functioning, feeling good, pain, fatigue, morning tiredness, stiffness, depression, anxiety, job ability and days of work missed. The total scores range from 0 to 100, with higher scores indicating more severe fibromyalgia-related symptoms and dysfunction. The severity levels of the fibromyalgia impact based on FIQ scores were categorized and proposed by Bennet [20] and Bennet et al. [21]. The cut-off points are: 0-58, mild fibromyalgia; 59-69, moderate fibromyalgia; 70-100, severe fibromyalgia.

8. Patient self-report assessment of fibromyalgia symptoms

The widespread pain index (WPI) and the symptom severity scale (SSS) are the most important patient self-report diagnostic variables based on ACR 2010c [10]. The WPI evaluates the number of areas in which the patient has had pain over the last week, with a score between 0 and 19 [10]. SSS measures the severity level of the following symptoms: fatigue, waking up unrefreshed, cognitive symptoms and the extent of somatic symptoms, which encapsulates a total of 41 different somatic symptoms including headache, pain or cramps in the lower abdomen and depression. The SSS score is between 0 and 12 , and a higher score indicates more severe fibromyalgiarelated symptoms [10].

The sum of the WPI and SSS generates the fibromyalgia severity (FS) scale [22], previously called the polysymptomatic distress scale (PDS) [23]. The FS scale ranges from 0 to 31 , and higher scores indicate worse fibromyalgia severity [22].

\section{Statistical Analysis}

All analyses were performed using SAS 8.2 software. For continuous variables, values with normal distributions are presented as mean and standard deviation (SD), while those with nonnormal distributions are presented as a median value and quartile deviation $(\mathrm{QR}=$ upper quartile median value-lower quartile median value). Frequency values of categorical variables were indicated as a percent (\%). The comparable data were analyzed with an independent sample $t$-test for continuous variables of normal 
distributions, chi-square test for continuous variables of non-normal distributions and frequency analysis and Mann-Whitney test for ranked data. Any $p$ value $<0.05$ was considered statistically significant.

\section{RESULTS}

\section{Demographic Characteristics of Patients}

A total of 124 Chinese patients diagnosed with fibromyalgia using ACR 1990c were recruited to this study. The mean of tender points counted (TPC) by physical examination of this cohort was $14.2,14.4$ for females and 13.1 for males, and there was no significant gender difference. Only 13 patients $(10.5 \%)$ had been previously diagnosed with fibromyalgia among these patients. The remaining 111 patients (89.5\%), who had visited at least one physician for their fibromyalgia-related symptoms prior to this study, were never given the diagnosis of fibromyalgia.

The demographic and medical histories among those 124 patients are shown in Table 1. All subjects in this cohort met the ACR 1990 criteria, with an average (SD) TPC count of 14.2 (3.0). The majority of the study subjects were women $(107,86.3 \%)$, married (78.2\%) and had high school or above education (86.3\%), and the female-to-male ratio was $6.3: 1$. The mean age of the cohort was 49.4 (range, 25-76) years. Males were significantly younger than females (43.6 vs. $50.1, p=0.027$ ). More male patients used tobacco $(p<0.001)$ and alcohol $(p<0.001)$ and had stable working conditions (either employed or retired, $p=0.016$ ); the men had higher incomes $(p=0.011)$ compared to their female counterparts.

The median duration of symptoms was 24 months with no significant gender differences; $45.2 \%$ patients had precipitating factors and $81.5 \%$ had aggravating factors for fibromyalgia. Forty-nine percent (49.2\%) of patients had received treatment for their symptoms, including pharmaceutical and/or Traditional Chinese Medicine (TCM) treatments. The most frequently used medication was sedatives (31.5\%), followed by NSAIDs (21.0\%), antidepressants
$(4.8 \%)$, pregabalin $(4.0 \%)$, amitriptyline $(2.4 \%)$ and duloxetine (1.6\%). The most frequently used TCM was herbal medicine $(21.8 \%)$, followed by topical medicinal paste (15.3\%), acupuncture/moxibustion (9.7\%) and acupotome $(4.0 \%)$. There was no significant gender difference for treatment types, except significantly more women (35.5\%) used sedatives than men $(5.9 \%)(p=0.015)$.

Most patients (76.6\%) had co-existing medical conditions, which affected women significantly more than men $(79.4 \%$ vs. $58.8 \%$, $p=0.019)$. The most frequent comorbidity in patients was cardiovascular diseases (25.0\%), followed by osteoarthritis/osteoporosis (18.5\%), tumors $(12.1 \%)$, ovarian cyst/adenomyosis $(8.9 \%)$, migraine $(7.3 \%)$, respiratory diseases $(6.5 \%)$, depression (5.6\%), irritable bowel syndrome $(4.0 \%)$ and temporomandibular arthritis (3.0\%). No significant gender difference in the comorbidity types was noted except that more men than women $(11.8 \%$ vs. $2.8 \%, p=0.018)$ had irritable bowel syndrome.

\section{Symptom Severity, Fibromyalgia Impact on Health Status and QOL}

The severity of five major symptoms (pain, fatigue, sleep quality, depression, stress) and the impact of fibromyalgia in patients of this cohort were analyzed and are shown in Table 2.

Among the five core symptoms of fibromyalgia, the majority of Chinese fibromyalgia patients experienced moderate to severe pain $(69.4 \%)$, severe fatigue $(70.2 \%)$, mild to moderate levels of depression (54.9\%) and stress (98.4\%). Only a small portion of patients had poor quality sleep (19.4\%), severe depression $(20.2 \%)$ or severe stress $(1.6 \%)$. No gender difference was observed.

For the impact of fibromyalgia on the health status of Chinese patients, according to the FIQ total score, the majority of the patients $(72.6 \%)$ were mildly affected by having this disease, while the health status of $19.4 \%$ patients was moderately affected, and only $8.1 \%$ were severely affected. Although there was no statistical difference, more men (17.6\%) seemed to be affected more severely by fibromyalgia than 
Table 1 Demographic and medical history of Chinese patients with fibromyalgia

\begin{tabular}{|c|c|c|c|c|}
\hline Characteristics & $\begin{array}{l}\text { Cohort total } \\
(n=124)\end{array}$ & $\begin{array}{l}\text { Female } \\
(n=107)\end{array}$ & $\begin{array}{l}\text { Male } \\
(n=17)\end{array}$ & $P$ value \\
\hline $\mathrm{TPC}^{\mathrm{a}}($ mean $(\mathrm{SD}))$ & $14.2(3.0)$ & $14.4(2.9)$ & $13.1(3.0)$ & 0.09 \\
\hline Age (years, mean $(S D))$ & $49.4(10.8)$ & $50.1(10.3)$ & $43.6(12.9)$ & 0.027 \\
\hline Han ethnicity $(n(\%))$ & $111(89.5)$ & $94(87.9)$ & $17(100.0)$ & 0.13 \\
\hline BMI (mean (SD)) & $23.3(3.0)$ & $23.2(3.1)$ & $23.6(2.5)$ & 0.57 \\
\hline Current tobacco use $(n(\%))$ & $5(4.0)$ & $1(1.0)$ & $4(23.5)$ & $<0.001$ \\
\hline Current alcohol use $(n(\%))$ & $4(3.2)$ & $0(0.0)$ & $4(23.5)$ & $<0.001$ \\
\hline Married (n (\%)) & $97(78.2)$ & $82(76.6)$ & $15(88.2)$ & 0.28 \\
\hline Employment $(n(\%))$ & & & & 0.016 \\
\hline Employed & $53(42.7)$ & $40(37.4)$ & $13(76.5)$ & \\
\hline Homemaker & $8(6.5)$ & $8(7.5)$ & $0(0.0)$ & \\
\hline Retired & $56(45.2)$ & $52(48.6)$ & $4(23.5)$ & \\
\hline Unemployed & $7(5.6)$ & $7(6.5)$ & $0(0.0)$ & \\
\hline Education $(n(\%))$ & & & & 0.19 \\
\hline Grades $1-9$ & $15(12.1)$ & $14(13.1)$ & $1(5.9)$ & \\
\hline Grades $10-12 /$ technical & $38(30.6)$ & $35(32.7)$ & $3(17.6)$ & \\
\hline College/university & $69(55.6)$ & $56(52.3)$ & $13(76.5)$ & \\
\hline Unknown & $2(1.6)$ & $2(1.9)$ & $0(0.0)$ & \\
\hline Household monthly income ${ }^{\mathrm{b}}(\mathrm{RMB}$, mean $(\mathrm{SD}))$ & & & & 0.011 \\
\hline $1000-2000$ & $8(6.7)$ & $8(7.5)$ & $0(0.0)$ & \\
\hline $2000-5000$ & $37(31.1)$ & $35(32.7)$ & $2(11.8)$ & \\
\hline $5000-10,000$ & $46(38.7)$ & $40(37.4)$ & $6(35.3)$ & \\
\hline Above 10,000 & $28(23.9)$ & $19(17.8)$ & $9(52.9)$ & \\
\hline Unknown & $5(4.0)$ & $4(3.7)$ & $0(0.0)$ & \\
\hline Residency in Beijing $(n(\%))$ & $96(77.4)$ & $81(75.7)$ & $15(88.2)$ & 0.24 \\
\hline Symptom duration, months (median $(\mathrm{Qd}))$ & $24(11.9)$ & $24(48.0)$ & $36(36.0)$ & 0.51 \\
\hline Having precipitating factors for FM symptoms $(n(\%))$ & $56(45.2)$ & $48(44.9)$ & $8(47.1)$ & 0.80 \\
\hline Having aggravating factors for FM symptoms $(n(\%))$ & $101(81.5)$ & $87(81.3)$ & $14(82.4)$ & 0.75 \\
\hline Previous FM treatment history $(\mathrm{n}(\%))$ & $61(49.2)$ & $51(47.7)$ & $10(58.2)$ & 0.58 \\
\hline \multicolumn{5}{|l|}{ Pre-study FM treatment means $(n(\%))$} \\
\hline Pregabalin & $5(4.0)$ & $4(3.7)$ & $1(5.9)$ & 1.00 \\
\hline Duloxetine & $2(1.6)$ & $2(1.9)$ & $0(0.0)$ & 1.00 \\
\hline Amitriptyline & $3(2.4)$ & $3(2.8)$ & $0(0.0)$ & 1.00 \\
\hline
\end{tabular}


Table 1 continued

\begin{tabular}{|c|c|c|c|c|}
\hline Characteristics & $\begin{array}{l}\text { Cohort total } \\
(n=124)\end{array}$ & $\begin{array}{l}\text { Female } \\
(n=107)\end{array}$ & $\begin{array}{l}\text { Male } \\
(n=17)\end{array}$ & $P$ value \\
\hline Venlafaxine & $6(4.8)$ & $5(4.7)$ & $1(5.9)$ & 1.00 \\
\hline NSAIDs & $26(21.0)$ & $23(21.5)$ & $3(17.6)$ & 0.77 \\
\hline Sedatives & $39(31.5)$ & $38(35.5)$ & $1(5.9)$ & 0.015 \\
\hline Acupuncture and moxibustion & $12(9.7)$ & $12(11.2)$ & $0(0.0)$ & 0.22 \\
\hline Chinese medicine & $27(21.8)$ & $25(23.4)$ & $2(11.8)$ & 0.52 \\
\hline Acupotome $^{c}$ & $5(4.0)$ & $4(3.7)$ & $1(5.9)$ & 1.00 \\
\hline Medicinal paste & $19(15.3)$ & $18(16.8)$ & $1(5.9)$ & 0.31 \\
\hline Coexisting illness $(n(\%))$ & $95(76.6)$ & $85(79.4)$ & $10(58.8)$ & 0.019 \\
\hline \multicolumn{5}{|l|}{ Coexisting illness types $(n(\%))$} \\
\hline Cardiovascular diseases & $31(25.0)$ & $27(25.2)$ & $3(17.6)$ & 0.56 \\
\hline Osteoarthrosis/osteoporosis & $23(18.5)$ & $21(19.6)$ & $2(11.8)$ & 0.53 \\
\hline Tumor/cancer & $15(12.1)$ & $13(12.1)$ & $2(11.8)$ & 1.00 \\
\hline Ovarian cyst/adenomyosis & $11(8.9)$ & $11(10.3)$ & $0(0.0)$ & 0.23 \\
\hline Respiratory diseases & $8(6.5)$ & $5(4.7)$ & $2(11.8)$ & 0.25 \\
\hline Depression & $7(5.6)$ & $7(6.5)$ & $0(0.0)$ & 0.59 \\
\hline Migraine & $9(7.3)$ & $9(8.4)$ & $0(0.0)$ & 0.36 \\
\hline Irritable bowel syndrome & $5(4.0)$ & $3(2.8)$ & $2(11.8)$ & 0.018 \\
\hline Temporomandibular arthritis & $2(3.0)$ & $1(0.9)$ & $1(5.9)$ & 0.26 \\
\hline
\end{tabular}

$B M I$ body mass index, $R M B$ renminbi, $Q d$ quartile deviation, $T P C$ tender point count by physical examination, $F M$ fibromyalgia, NSAIDs nonsteroidal antiinflammatory drugs

a The TPC is a part of the clinical examination for fibromyalgia ranging from 0 to 18 , with higher values indicating a lower pain threshold

${ }^{b}$ One RMB = 1 yuan, and during most of 2018, 1000 RMB (yuan) was equivalent to US\$ 147.71

c An acupuncture operation using an instrument shaped like a needle knife

women $(6.5 \%)$ as indicated by the FIQ severity grade. For the health-related QOL, the mental health of men was affected more than that of women, with lower scores for SF-36 MCS $(p=0.043)$ and SF-36 subscales of role emotional $(p=0.006)$. Females had more painful sites, with higher WPI counts than their male counterparts $(11.1 \quad(4.7 \%)$ vs. $8.6 \quad(3.9 \%)$, $p=0.038)$. No gender difference was observed on the impact of fibromyalgia on the SSS and FS in these Chinese patients.

In short, most Chinese patients' health was mildly or moderately affected by fibromyalgia.
Fibromyalgia affected the QOL more negatively in relation to the physical than mental health of these patients, and men had a more profound impact on mental health and reported fewer painful sites compared to women.

\section{Exhibition of Clinical Features of Fibromyalgia between Chinese Subjects and Those of Other Countries}

To show the characteristics and impact of fibromyalgia among fibromyalgia patients from 
Table 2 Clinical features and symptom severity of Chinese fibromyalgia patients

\begin{tabular}{|c|c|c|c|c|}
\hline Characteristics & Cohort $(N=124)$ & Female $(N=107)$ & Male $(N=17)$ & $P$ value \\
\hline Pain VAS score ${ }^{\mathrm{a}}($ mean $(\mathrm{SD}))$ & $55.9(22.2)$ & $56.2(21.7)$ & $54.1(25.5)$ & 0.72 \\
\hline NRS $\operatorname{grade}^{\mathrm{b}}(n(\%))$ & & & & 0.20 \\
\hline NRS-Mild pain & $38(30.6)$ & $31(29.0)$ & $7(41.2)$ & \\
\hline NRS-Moderate pain & $56(45.2)$ & $52(48.6)$ & $5(29.4)$ & \\
\hline NRS-Severe pain & $30(24.2)$ & $24(22.4)$ & $5(29.4)$ & \\
\hline MAF-GFI score $^{c}$ (mean (SD) & $38.4(8.9)$ & $33.7(8.6)$ & $31.8(10.7)$ & 0.43 \\
\hline MAF-GFI grade ${ }^{c}(n(\%))$ & & & & 1.00 \\
\hline Severe fatigue & $87(70.2)$ & $75(70.1)$ & $12(70.6)$ & \\
\hline Fatigue & $37(29.8)$ & $32(29.9)$ & $5(29.4)$ & \\
\hline PSQI score ${ }^{\mathrm{d}}($ mean $(\mathrm{SD}))$ & $11.0(4.2)$ & $10.9(4.0)$ & $11.5(5.3)$ & 0.69 \\
\hline PSQI grade ${ }^{\mathrm{d}}(n(\%))$ & & & & 0.17 \\
\hline Better sleep & $11(8.9)$ & $8(7.5)$ & $3(17.6)$ & \\
\hline Good sleep & $47(37.9)$ & $43(40.2)$ & $5(29.4)$ & \\
\hline Fair sleep & $42(33.9)$ & $38(35.5)$ & $4(23.5)$ & \\
\hline Very poor sleep & $24(19.4)$ & $18(16.8)$ & $5(29.4)$ & \\
\hline BDI-II score $^{\mathrm{e}}($ mean $(\mathrm{SD}))$ & $9.4(6.4)$ & $9.7(6.5)$ & $7.5(5.9)$ & 0.21 \\
\hline BDI-II grade ${ }^{\mathrm{e}}(n(\%))$ & & & & 0.50 \\
\hline No depression & $31(25.0)$ & $25(23.4)$ & $6(35.3)$ & \\
\hline Mild depression & $27(21.8)$ & $22(20.6)$ & $5(29.4)$ & \\
\hline Moderate depression & $41(33.1)$ & $37(34.6)$ & $4(23.5)$ & \\
\hline Severe depression & $25(20.2)$ & $23(21.5)$ & $2(11.8)$ & \\
\hline PSS score ${ }^{f}($ mean $(S D))$ & $25.0(9.4)$ & $24.9(9.4)$ & $25.2(9.4)$ & 0.91 \\
\hline PSS $\operatorname{grade}^{\mathrm{f}}(n(\%))$ & & & & 0.81 \\
\hline Mild stress & $78(62.9)$ & $67(62.6)$ & $11(64.7)$ & \\
\hline Moderate severe stress & $44(35.5)$ & $39(36.4)$ & $6(35.3)$ & \\
\hline Severe stress & $2(1.6)$ & $2(1.9)$ & $0(0.0)$ & \\
\hline FIQ total score ${ }^{\mathrm{g}}($ mean $(\mathrm{SD}))$ & $49.7(15.1)$ & $49.0(15.0)$ & $54.7(15.0)$ & 0.16 \\
\hline FIQ $\operatorname{grade}^{\mathrm{g}}(n(\%))$ & & & & 0.24 \\
\hline Severe & $10(8.1)$ & $7(6.5)$ & $3(17.6)$ & \\
\hline Moderate & $24(19.4)$ & $21(19.6)$ & $3(17.6)$ & \\
\hline Mild & $90(72.6)$ & $79(73.8)$ & $11(64.7)$ & \\
\hline \multicolumn{5}{|l|}{ SF-36 ${ }^{\mathrm{h}}($ mean $(\mathrm{SD}))$} \\
\hline Physical functioning & $60.9(23.0)$ & $60.5(22.4)$ & $63.2(27.0)$ & 0.65 \\
\hline
\end{tabular}


Table 2 continued

\begin{tabular}{lllll}
\hline Characteristics & Cohort $(\boldsymbol{N}=\mathbf{1 2 4})$ & Female $(\boldsymbol{N}=\mathbf{1 0 7})$ & Male $(\boldsymbol{N}=\mathbf{1 7})$ & $\boldsymbol{P}$ value \\
\hline Role physical & $27.0(35.4)$ & $27.3(36.1)$ & $25.0(31.9)$ & 0.80 \\
Pain index & $39.7(17.8)$ & $39.3(17.8)$ & $41.8(18.1)$ & 0.61 \\
General health perceptions & $28.7(17.3)$ & $28.7(17.1)$ & $28.5(18.9)$ & 0.97 \\
Vitality & $34.6(19.3)$ & $34.8(19.1)$ & $33.5(20.8)$ & 0.80 \\
Social functioning & $56.9(24.9)$ & $57.9(24.7)$ & $50.0(25.4)$ & 0.22 \\
Role emotional & $51.3(45.3)$ & $55.1(45.8)$ & $27.5(33.8)$ & 0.006 \\
Mental health index & $47.2(23.0)$ & $48.2(22.5)$ & $40.6(25.9)$ & 0.21 \\
PCS & $32.5(16.7)$ & $32.5(16.7)$ & $32.2(16.7)$ & 0.94 \\
MCS $_{\text {WPI }}^{\text {i }}$ (mean (SD)) & $48.8(20.9)$ & $50.4(21.0)$ & $39.3(18.5)$ & 0.043 \\
SSS $^{\text {j }}$ (mean (SD)) & $10.8(4.7)$ & $11.1(4.7)$ & $8.6(3.9)$ & 0.038 \\
FS $^{\mathrm{k}}$ (mean (SD)) & $7.6(2.5)$ & $7.4(2.6)$ & $8.7(1.8)$ & 0.06 \\
\hline
\end{tabular}

MAF-GFI Global Fatigue Index of the Multidimensional Assessment of Fatigue, PSQI Scores on the Pittsburgh Sleep Quality Index; BDI-II Beck II Depression Inventory, PSS Perceived Stress Scale, FIQ Fibromyalgia Impact Questionnaire, SF-36 Short Form-36 Health Status Questionnaire, PCS physical component score, MCS mental component score, WPI widespread pain index, SSS symptom severity scale, FS fibromyalgia severity scale

${ }^{a}$ Pain VAS is a measurement for body pain. Scores range from 0 to $100 \mathrm{~mm}$, with higher scores indicating greater pain

b The NRS allows a person to describe the intensity of his/her pain as a number ranging from 0 to $10.0=$ no pain, $1-3=$ mild pain, 4-6 = moderate pain, 7-9 = severe pain and $10=$ bad as it could be

${ }^{c}$ MAF-GFI measures fatigue severity scoring from 0 to 50 , with higher scores indicating greater fatigue severity. MAF-GFI score of 30 points was used to define clinically severe fatigue

d PSQI measures sleep quality. Scores on the PSQI range from 0 to 21, with higher scores indicating worse sleep quality. The cut-off scores for PSQI are: 1-5 points = "very little compromise of sleep quality," 6-10 = "good sleep quality," 11-15 = "fair sleep quality" and 16-21 = "very poor sleep quality"

${ }^{\mathrm{e}}$ BDI-II assesses the severity of depressive symptoms. Scores range from 0 to 39, with higher scores indicating a greater degree of depression severity. The cut-off scores for BDI-II are: $0-4$ points $=$ "no relevant depressive symptoms," $5-7=$ "mild depressive symptoms," 8-15 = "moderate depressive symptoms" and 16-39= "severe depressive symptoms"

${ }^{f}$ PSS measures the perception of stress and current levels of experienced stress. Scores range from 0 to 56, with higher scores indicating a greater degree of symptom severity. The cut-off scores for PSS are: 0-28 points = "mild stress," 29-42= "moderately severe stress" and 43-56 = "severe stress"

g FIQ assesses fibromyalgia symptoms and function domains. Scores range from 0 to 100, with higher scores indicating more severe symptoms. The cut-off scores for FIQ are: 0-58 points = "mild fibromyalgia," 59-69 points = "moderate fibromyalgia" and 70-100 = "severe fibromyalgia"

h SF-36 is a self-administered, 36-item questionnaire that assesses the physical and mental quality of life. The physical and mental component summaries can be combined ranging from 0 to 100 , with higher scores indicating better health status ${ }^{\mathrm{i}}$ The WPI is a part of 2010c and 2016c that measures the number of body pain points ranging from 0 to 19 , with higher scores indicating more pain points

j The SSS is a part of 2010c and 2016c that evalutes fibromyalgia symptom severity ranging from 0 to 12 , with higher scores indicating more pain. The SSS includes four parts (in italics): the fatigue, waking unrefreshed and cognitive symptom severity as well as severity of somatic symptoms

${ }^{\mathrm{k}}$ The FS is the sum of the WPI and SSS, which is also called the polysymptomatic distress scale, and ranges from 0 to 31 with higher scores indicating worse fibromyalgia severity 
China and other countries, data from this study and seven previously published studies (two Chinese studies [3, 24], one Japanese study [25], one Korean study [26], one European study [27] and two US American studies [10, 28]) are presented in Table 3. In this study, the mean age of patients (49.4 years) was similar to those of other studies, ranging from 47.3 to 54.6 years, with the exception of a much younger cohort with mean age of 37 years in Zhang's Chinese study [24]. The gender (male-to-female) ratio of this study was $1: 6.3$, comparable to those from a Korean study (1:6.7) [26] and Wolfe's US study (1:5.9) [10], but lower than those from two efficacy studies, i.e., Ohta's Japanese study (1:8.1) [25] and Branco's European study (1:27.5) [27], and one cross-sectional study with referral patients in Jiao's US study (1:16.7) [28]. The TPC counts of this study (14.2) were comparable to those of other countries $[3,10,26,28]$, ranging from 12.9 to 16.2 . The TPC count in Zhang's Chinese study was 9.7, which was due to the ACR 2010 criteria used to recruit patients in their study.

According to the WPI, SSS, FS, Pain VAS and BDI-II scores, Chinese fibromyalgia patients were less likely to have severe fibromyalgia symptoms, their daily function was less likely to be impacted by fibromyalgia (as shown in FIQ), and they might have a better QOL, especially better mental health, when considering the manifestations of patients from other countries.

\section{DISCUSSION}

Compared to the $2-5 \%$ prevalence rate of fibromyalgia in other countries, current studies indicate that the prevalence of fibromyalgia in the Chinese population is much lower, ranging from 0.03 to $0.12 \%$ in Mainland China [4-6] and $0.82 \%$ in Hong Kong China [3]. If these limited studies underestimate the prevalence of fibromyalgia in China, possible explanations include low awareness of the disease by Chinese health providers, different clinical manifestations of the disease in Chinese populations or different practices in applying the diagnostic criteria for fibromyalgia in China. The aim of this study was to explore these unanswered questions.

In Mainland China, most of the population does not know about fibromyalgia, and awareness among health care providers is also poor. Prior survey studies showed that nearly $30 \%$ of Chinese rheumatologists believed that fibromyalgia was a psychological disorder, < $20 \%$ of rheumatologists have ever knowingly treated a patient with fibromyalgia [29], and > $50 \%$ of the rheumatologists did not know the pathogenesis of fibromyalgia [30]. Due to lack of the knowledge about fibromyalgia, physicians often miss the diagnosis, do not consider it a legitimate medical disorder and/or assess and treat symptoms without a formal diagnosis [30]. The current study indicates that of the participants who visited a specialty clinic for fibromyalgia-related symptoms at least once prior to being enrolled in this study, $90 \%$ of them failed to receive the diagnosis of fibromyalgia. These findings suggest that the prevalence of fibromyalgia may be underestimated in China, in part due to lack of knowledge about the disease.

Past study has shown that demographic predictors of fibromyalgia include age, female gender, marriage status and employment history, among others [31].

The average age of the patients in this study was 49.4 years, which is similar to those in other studies with different races, ranging from 47.3 years [25] to 54.6 years [10], with the exception of Zhang's Chinese fibromyalgia study in which the average age was 37 years [24]. One possible explanation for this discrepancy may be referral bias because study subjects were recruited from a military hospital that typically serves younger patients though the general public can visit the hospital as well.

Fibromyalgia predominantly affects females. In this study, $86 \%$ of the patients were female, which is consistent with other studies $[10,25-28,32]$. The male-to-female ratio in this study was $1: 6.3$, which is similar to the $1: 5.9$ to 6.8 reported for US patients with fibromyalgia $[10,32]$, but different from several other studies, especially from two other Chinese fibromyalgia studies [3, 24]. In this clinical study, the patients were recruited from a hospital serving 
Table 3 Comparison of clinical features of fibromyalgia between Chinese patients and patients of other race or ethnic groups

\begin{tabular}{|c|c|c|c|c|c|c|c|c|}
\hline Reference & Jiao & Scudds ${ }^{3}$ & Zhang $^{24 a}$ & Wolfe $^{10 b}$ & Ohta $^{25}$ & $\mathrm{Kim}^{26}$ & Branco $^{27}$ & $\mathrm{Jiao}^{28}$ \\
\hline Country of research & $\begin{array}{l}\text { Beijing, } \\
\text { China }\end{array}$ & $\begin{array}{l}\text { Hong Kong, } \\
\text { China }\end{array}$ & $\begin{array}{l}\text { Beijing, } \\
\text { China }\end{array}$ & USA & Japan & Korea & Europe & USA \\
\hline Date of publication & $\begin{array}{l}\text { This } \\
\text { report }\end{array}$ & 2006 & 2017 & 2010 & 2012 & 2012 & 2010 & 2015 \\
\hline ACR criteria & 1990 & 1990 & 2010 & 1990 & 1990 & 1990 & 1990 & 1990 \\
\hline No. of patients & 124 & 7 & 107 & 196 & 498 & 77 & 876 & 939 \\
\hline Age (mean (SD)) & $49.4(10.8)$ & $50.1(15.6)$ & 37 (NA) & $\begin{array}{l}54.6 \\
\quad(12.9)\end{array}$ & $\begin{array}{l}47.3 \\
(12.3)\end{array}$ & $\begin{array}{l}49.3 \\
\quad(1.3)\end{array}$ & $\begin{array}{l}48.8 \\
\quad(9.8)\end{array}$ & $\begin{array}{l}48.6 \\
\quad(12.9)\end{array}$ \\
\hline Female gender $(n(\%))$ & $107(86.3)$ & $4(57.1)$ & $86(80.4)$ & $\begin{array}{l}236 \\
\quad(91.8)\end{array}$ & $\begin{array}{l}443 \\
(89.0)\end{array}$ & $\begin{array}{l}67 \\
(87.0)\end{array}$ & $\begin{array}{l}826 \\
(94.3)\end{array}$ & $\begin{array}{l}886 \\
(94.4)\end{array}$ \\
\hline Male-to-female ratio & $1: 6.3$ & $1: 1.3$ & $1: 4.1$ & $1: 5.9$ & $1: 8.1$ & $1: 6.7$ & $1: 27.5$ & $1: 16.7$ \\
\hline $\operatorname{TPC}(n(\%))$ & $14.2(3.0)$ & $12.9(1.9)$ & $9.7(5.2)$ & $\begin{array}{l}15.9 \\
(2.3)\end{array}$ & NA & $\begin{array}{l}13.7 \\
(0.3)\end{array}$ & $\mathrm{NA}$ & $\begin{array}{l}16.2 \\
(\mathrm{NA})\end{array}$ \\
\hline WPI (mean $(S D))$ & $10.6(4.7)$ & NA & $11.3(3.8)$ & $\begin{array}{l}11.4 \\
(4.4)\end{array}$ & NA & $\begin{array}{l}10.9 \\
(0.5)\end{array}$ & NA & NA \\
\hline SSS (mean (SD)) & $7.5(2.5)$ & NA & $7.9(2.1)$ & $8.0(2.6)$ & NA & $\begin{array}{l}8.4 \\
\quad(0.3)\end{array}$ & NA & NA \\
\hline FS (mean $(\mathrm{SD}))$ & $18.1(5.7)$ & NA & $19.2(4.4)$ & $\begin{array}{l}19.1 \\
(\mathrm{NA})\end{array}$ & NA & NA & NA & NA \\
\hline $\begin{array}{l}\text { Pain VAS score (mean } \\
\quad(\mathrm{SD}))\end{array}$ & $55.9(22.2)$ & $40.3(15.3)$ & $42.7(18.1)$ & $\begin{array}{l}65.0 \\
\quad(23.0)\end{array}$ & $\begin{array}{l}67.7 \\
\quad(9.6)\end{array}$ & $\begin{array}{l}76.0 \\
(1.0)\end{array}$ & $\begin{array}{l}65.2 \\
(12.8)\end{array}$ & NA \\
\hline $\begin{array}{l}\text { BDI-II score (mean } \\
\quad(\mathrm{SD}))\end{array}$ & $9.4(6.4)$ & NA & NA & NA & NA & NA & $\begin{array}{l}10.6 \\
(6.7)\end{array}$ & NA \\
\hline $\begin{array}{l}\text { FIQ total score (mean } \\
\quad(\mathrm{SD}))\end{array}$ & $49.7(15.1)$ & NA & NA & NA & $\begin{array}{l}52.2 \\
(15.2)\end{array}$ & $\begin{array}{l}75.8 \\
(2.2)\end{array}$ & $\begin{array}{l}56.9 \\
(11.9)\end{array}$ & $\begin{array}{l}62.9 \\
(\mathrm{NA})\end{array}$ \\
\hline $\begin{array}{l}\text { SF-36 PCS (mean } \\
\quad(\mathrm{SD}))\end{array}$ & $32.5(16.7)$ & NA & 40.5 (NA) & NA & NA & NA & $\begin{array}{l}33.6 \\
(6.8)\end{array}$ & $\begin{array}{l}26.8 \\
(\mathrm{NA})\end{array}$ \\
\hline $\begin{array}{l}\text { SF-36 MCS (mean } \\
\quad(\text { SD)) }\end{array}$ & $48.8(20.9)$ & NA & 40.5 (NA) & NA & NA & NA & $\begin{array}{l}46.6 \\
(9.8)\end{array}$ & $\begin{array}{l}39.9 \\
\text { (NA) }\end{array}$ \\
\hline
\end{tabular}

ACR American College of Rheumatology, TPC tender point count by physical examination, WPI Widespread Pain Index, SSS Symptom Severity Scale, FS Fibromyalgia Severity Scale, VAS visual analog scale, MAF-GFI Global Fatigue Index of the Multidimensional Assessment of Fatigue, PSQI Pittsburgh Sleep Quality Index scores, BDI-II Beck II Depression Inventory, PSS Perceived Stress Scale, FIQ = Fibromyalgia Impact Questionnaire, SF-36 Short Form-36 Health Status Questionnaire, $P C S$ physical component score, $M C S$ mental component score, $N A$ not available, means studies did not evaluate the specific symptom, except for using Multidimensional Fatigue Inventory (MFI) instead of the MAF-GFI in Branco's study

a The scores of the SF-36 PCS and MCS in Zhang's report were based on a smaller cohort of 86

b The age and female gender in Wolfe's report were based on a larger cohort of 258 
the general public in Beijing, China. Zhang's fibromyalgia study [24] was also conducted in Beijing but the patients were recruited from a military hospital, which has predominantly male patients. The relatively high male-to-female ratio, 1:4.1 in Zhang's study, therefore, might be the results of the study's referral bias. In Roger's Hong Kong fibromyalgia study [3], the male-to-female ratio was $1: 1.3$, which is much higher than what this and Zhang's study found. The reason for such a relatively high male ratio might stem from the small sample size, as Zhang's study was an epidemiology study and only seven fibromyalgia patients were diagnosed, from which the gender ratio was generalized. On the other hand, the male-tofemale ratio of this study is lower than those in some other clinical studies, i.e., at 1:8.1 and $1: 27.5$, respectively, in two efficacy studies $[25,27]$ and at $1: 16.7$ in a cross-sectional study with treatment referral patients [28]. The explanation for such gender ratio differences might be the inclusion criteria used for those studies.

Marriage was a negative predictor of fibromyalgia [31]. In this study, the marriage rate $(78.2 \%)$ of the patients was higher than those reported in a US study [28]. These differences might partially result from the culture in China. For Chinese people of this generation, aged 50 years (average age of 49.4 in this study) and above, the divorce rate is low as divorce is socially stigmatized.

In the current study, the average employment rate $(42.7 \%)$ was low and the retirement rate $(45.2 \%)$ was high compared to the rates reported in other countries [28]. With an average age of 50.1 years, only $37.4 \%$ of females were employed, less than half of the employment rate of males $(76.5 \%)$. In contrast, the female retirement rate was twice that of males, $48.6 \%$ vs. $23.5 \%$. Such differences at a relatively young age (average age of 49.4 years) may be less likely because of the disease impact but rather caused by China's retirement policy, in which women who are general workers retire at 50 years old and professionals at 55, and men retire at 60 years old in general.

In this cohort, the median duration of having fibromyalgia symptoms was 24 months, which indicated that the disease duration of these Chinese fibromyalgia patients was shorter than that in most of the studies in other countries $[25,27,28]$. In addition, fewer Chinese patients reported a treatment history for their fibromyalgia symptoms than patients from other countries [33], as only an half of the patients in this study had sought additional treatment for their fibromyalgia symptoms. The most commonly used treatments were various forms of Chinese medicine, including herbal medicine, medicinal paste/patch, acupuncture and moxibustion, and acupotome, in descending order. The most frequently used medications were sedatives $(31.5 \%)$, followed by NSAIDs (21.0\%), antidepressants (4.8\%), pregabalin $(4.0 \%)$, amitriptyline $(2.4 \%)$ and duloxetine $(1.6 \%)$. The finding that only a few patients were treated with medication typically prescribed to treat fibromyalgia in western and developed countries supports the hypothesis that fibromyalgia awareness in China is low.

Chinese fibromyalgia patients in this cohort also exhibited medical comorbidities described in studies from other countries [34, 35]. The most frequent comorbidity was cardiovascular disease, followed by osteoarthritis/osteoporosis, tumor, ovarian cyst/adenomyosis, migraine, respiratory diseases, depression, irritable bowel syndrome, and temporomandibular arthritis, in descending order. No gender difference was found in the types of comorbidities with the exception that more men had irritable bowel syndrome (IBS) than women $(11.8 \%$ vs. $2.8 \%)$. While the gender predilection of IBS was statistically significant, only five patients (4.0\%) had IBS in this cohort. Thus, whether this finding is generalizable or if it is clinically significant should be further investigated.

In this study, patients' fibromyalgia symptoms and their severity were examined using various questionnaires that assess pain, fatigue, sleep, depression, stress, fibromyalgia impact and health-related QOL. In this cohort, patients suffered from various degrees of fibromyalgia symptoms. There were no gender differences between these symptoms, except male patients had significantly lower role emotional scores (27.5) in health-related QOL compared to that of females (55.1) as well as lower mental 
component scores (39.3) than females (50.4). The cause for such gender differences was not clear but might relate to a greater number of males being employed and fibromyalgia compromising their mental health more profoundly.

When comparing the symptom severity of fibromyalgia of Chinese patients in this study to that of the patients from other countries, we found the following trends: (1) Chinese fibromyalgia patients seemed to have milder fibromyalgia-related symptoms, evidenced by lower Pain VAS scores, lower SSS scores and lower BDI-II (depression) scores and (2) the lives of Chinese fibromyalgia patients were less impacted by fibromyalgia than the patients of other races, as evidenced by lower FIQ scores. The explanation for such observations might be: (1) the relatively short duration of the disease of the patients, (2) the earlier retirement age for Chinese women, which reduced the physical and psychological stress of working with concurrent fibromyalgia, (3) the high marriage rate and stable family relationships, (4) the Chinese diet, which consists of more vegetables than meat, or (5) cultural differences having a substantial influence upon self-reporting pain and other health issues.

Limitations of this study include: (1) less assurance about our findings, especialy the gender comparison findings, due to the small sample size, (2) patients were recruited mainly from one city and (3) some of those employed instruments' Chinese validations are still pending (FIQ, MAF, WPI, SSS). Therefore, our research group has begun the necessary regional tasks of idiomatic translation and validation of many fibromyalgia diagnostic and outcome assessment instruments (ClinicalTrials.gov identifier: NCT03381131).

\section{CONCLUSIONS}

In this cross-sectional study, the characteristics of Chinese patients with fibromyalgia were likely to be demographically similar to those of fibromyalgia patients in clinical studies abroad, but their fibromyalgia-related symptoms might be milder and their mental QOL might be less affected by the disease compared to patients from other countries. We also found differences between male and female patients. Further cross-sectional studies with a larger sample size and nationwide study sites are needed to replicate the clinical findings of this fibromyalgia study group to ascertain if our findings are generalizable to the entire Chinese population and ultimately help improve the diagnosis and management of fibromyalgia in China.

\section{ACKNOWLEDGEMENTS}

We sincerely thank the participants of the study.

Funding. This research was supported by Beijing Municipal Science \& Technology Commission of China. (grant no. Z141107002514094 and Z181100001718153). The journal's Rapid Service Fee is funded by Guang'anmen Hospital.

Authorship. All named authors meet the International Committee of Medical Journal Editors (ICMJE) criteria for authorship for this article, take responsibility for the integrity of the work as a whole, and have given their approval for this version to be published.

Authorship Contributions. Juan Jiao and Quan Jiang conceived and designed the study. Juan Jiao drafted the paper. Juan Jiao, Quan Jiang and Yayun Zhao recruited the patients for this study. Juan Jiao and Yayun Zhao evaluated the participants and recorded information about them. Juan Jiao and Wen Wang analyzed the data. Drs. Cheng and Wang edited and revised the paper.

Disclosures. Juan Jiao, Zengyu Cheng,Wen Wang, Yayun Zhao and Quan Jiang have nothing to disclose.

Compliance with Ethics Guidelines. This study was approved by the Institutional Review Board (IRB) of the Guang'anmen Hospital (reference no. NCT02449395) and performed in accordance with the Helsinki Declaration of 
1975 and its later amendments or comparable ethical standards. The participants provided their written informed consent to participate in this study.

Data Availability. The datasets generated during and/or analyzed during the current study are available from the corresponding author on reasonable request.

Open Access. This article is licensed under a Creative Commons Attribution-NonCommercial 4.0 International License, which permits any non-commercial use, sharing, adaptation, distribution and reproduction in any medium or format, as long as you give appropriate credit to the original author(s) and the source, provide a link to the Creative Commons licence, and indicate if changes were made. The images or other third party material in this article are included in the article's Creative Commons licence, unless indicated otherwise in a credit line to the material. If material is not included in the article's Creative Commons licence and your intended use is not permitted by statutory regulation or exceeds the permitted use, you will need to obtain permission directly from the copyright holder. To view a copy of this licence, visit http:// creativecommons.org/licenses/by-nc/4.0/.

\section{REFERENCES}

1. Arnold LM, Clauw DJ, Dunegan LJ, Turk DC, Fibro C. A framework for fibromyalgia management for primary care providers. Mayo Clin Proc. 2012;87: 488-96.

2. White KP, Harth M. Classification, epidemiology, and natural history of fibromyalgia. Curr Pain Headache Rep. 2001;5(4):320.

3. Roger AS, Edmund KML, Rhonda JS. The prevalence of fibromyalgia syndrome in chinese people in Hong Kong. J Musculoskeletal Pain. 2006;14(2): 3-11.

4. Lin L, Xiao Z, Lin S, Chen R, Lin R, Du W. A rural population survey of soft tissue rheumatic pain in Shantou. China Shanxi Med J. 2007;36:678-80.
5. Zeng SY, Gong Y, Zhang YP, et al. Changes in the prevalence of rheumatic diseases in Shantou, China, in the past three decades: A COPCORD Study. PLoS ONE. 2015;10(9):e0138492.

6. Zang CZ, Li X, Dong H, Zhang A. Epidemiological study of rheumatic diseases in Taiyuan area. Chin Remd Clin (Chinese). 2007;7:597-602.

7. Kim SH, Bae GR, Lim HS. Prevalence and risk factors of fibromyalgia syndrome and chronic widespread pain in two communities in Korea-first report in Korean(Korean). J Korean Rheum Assoc. 2006;13(1): $18-25$.

8. Marques AP, Santo AS, Berssaneti AA, Matsutani LA, Yuan SL. Prevalence of fibromyalgia: literature review update. Rev Bras Reumatol 57 (4): 356-63.

9. Wolfe F, Smythe HA, Yunus MB, et al. The American College of Rheumatology 1990 Criteria for the Classification of Fibromyalgia. Report of the Multicenter Criteria Committee. Arthritis Rheum 1990;33:160-72.

10. Wolfe F, Clauw DJ, Fitzcharles MA, et al. The American College of Rheumatology of preliminary diagnostic criteria for fibromyalgia and measurement of symptom severity. ACR. 2010;62(5): 600-10.

11. Huskisson E. Visual analogue scale. Pain Mesurement \& Assessment. 1983:4349.

12. Numerical Rating Scale. Springer Berlin Heidelberg; 2007.

13. Belza BL, Henke CJ, Yelin EH, Epstein WV, Gilliss CL. Correlates of fatigue in older adults with rheumatoid arthritis. Nurs Res. 1993;42:93-9.

14. Belza BL. Comparison of self-reported fatigue in rheumatoid arthritis and controls. J Rheumatol. 1995;22:639-43.

15. Liu XC, Tang MQ, Hu L, et al. Reliability and validity of the Pittsburgh sleep quality index. Chin J Psychia (Chinese). 1996;29(2):103-7.

16. Zheng $\mathrm{H}$, Zheng Y. The Application of depression self-rating questionnaire (BDI) in depression patients [J]. Chin J Nerv and Ment (Chinese). 1987;04:236-7.

17. Yang TZ, Huang HT. An epidemiological study on stress among urban residents in social transition period. Chin J Epidemiol (Chinese). 2003;24(9): 760-4.

18. Li L, Wang H, Shen Y. Development and psychometric tests of a Chinese version of the SF-36 Health 
Survey Scales. Zhonghua Yu Fang Yi Xue Za Zhi (Chinese). 2002;36(2):109-13.

19. Burckhardt CS, Clark SR, Bennett RM. The fibromyalgia impact questionnaire: development and validation. J Rheumatol. 1991;18(5):728-33.

20. Bennet RM. The Fibromyalgia Impact Questionnaire (FIQ): a review of its development, current version, operating characteristics and uses. Clin Exp Rheumatol. 2005;23(Suppl. 39):S154-62.

21. Bennet RM, Bushmakin AG, Cappelleri JC, Zlateva G, Sadosky AB. Minimal clinically important difference in the fibromyalgia impact questionnaire. J Rheumatol. 2009;36:1304-11.

22. Wolfe F, Clauw DJ, Fitzcharles MA, et al. 2016 Revisions to the 2010/2011 fibromyalgia diagnostic criteria. Semin Arthritis Rheum. 2016;46(3):319-29.

23. Wolfe F, Brähler E, Hinz A, Häuser W. Fibromyalgia prevalence, somatic symptom reporting, and the dimensionality of polysymptomatic distress: results from a survey of the general population. Arthitis Care Res. 2013;65(5):777-85.

24. Zhang Y, Liang D, Jiang R, et al. Clinical, psychological features and QOL of fibromyalgia patients: a cross-sectional study of Chinese sample. Clin Rheumatol. 2018;37(2):527-37.

25. Ohta H, Oka H, Usui C, Ohkura M, Suzuki M, Nishioka K. A randomized, double-blind, multicenter, placebo-controlled phase III trial to evaluate the efficacy and safety of pregabalin in Japanese patients with fibromyalgia. Arthritis Res Ther. 2012;14(5):R217.

26. Kim SL, Lee SH, Kim HR. Applying the ACR Preliminary Diagnostic Criteria in the Diagnosis and Assessment of Fibromyalgia. Korean J Pain 25(3): 173-82.

27. Branco JC, Zachrisson O, Perrot S, Mainguy Y. Multinational Coordinator Study Group. A European multicenter randomized double-blind placebo-controlled monotherapy clinical trial of milnacipran in treatment of fibromyalgia. J Rheumatol. 2010;37(4):851-9.
28. Jiao J, Vincent A, Cha SS, Luedtke CA, Kim $\mathrm{CH}$, Oh TH. Physical trauma and infection as precipitating factors in patients with fibromyalgia [J]. Am J phys Med Rehabil. 2015;94(12):1075-82.

29. Mu R, Li C, Zhu JX, et al. National survey of knowledge, attitude and practice of fibromyalgia among rheumatologists in China[J]. Int J Rheum Dis. $2013 ; 16: 258-63$.

30. Jiao J, Zhang RM, Jiang Q. The cognitive level of fibromyalgia syndrome in rheumatologists of traditional chinese medicine. J Tradi Chin Medic (Chinese), 2017,58(20):1740-2,59.

31. Walitt B, Katz RS, Bergman MJ, Wolfe F. Threequarters of persons in the US population reporting a clinical diagnosis of fibromyalgia do not satisfy fibromyalgia criteria: The 2012 National Health Interview Survey. PLoS ONE. 2016;11(6):e0157235.

32. Wolfe F, Ross K, Anderson J, Russell IJ, Hebert L. The prevalence and characteristics of fibromialgia in the general population. Arthritits Rheum. 1995;38:19-28.

33. Mohabbat AB, Mahapatra S, Jenkins SM, Bauer BA, Vincent A, Wahner-Roedler DL. Use of complementary and integrative therapies by fibromyalgia patients: A 14-Year follow-up study. Mayo Clin Proc Innov Qual Outcomes. 2019;3(4):418-28.

34. Vincent A, Whipple MO, McAllister SJ, Aleman KM, St Sauver JL. A cross-sectional assessment of the prevalence of multiple chronic conditions and medication use in a sample of community-dwelling adults with fibromyalgia in Olmsted County, Minnesota. BMJ Open. 2015;5(3):e006681.

35. Weir PT, Harlan GA, Nkoy FL, et al. The incidence of fibromyalgia and its associated comorbidities: a population-based retrospective cohort study based on International Classification of Diseases, 9th Revision codes. J Clin Rheumatol. 2006;12:124-8.

36. Goren A, Mould-Quevedo J, daCosta Di Bonaventura M. Prevalence of pain reporting and associated health outcomes across emerging markets and developed countries. Pain Med. 2014;15(11): 1880-91. 